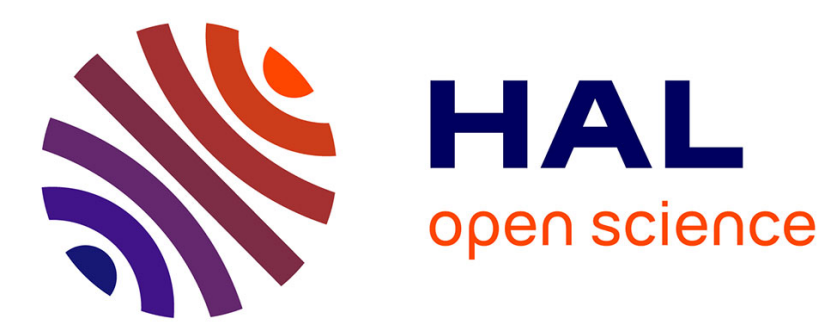

\title{
Interval State Observer for Nonlinear Time Varying Systems
}

Denis Efimov, Tarek Raissi, Stanislav Chebotarev, Ali Zolghadri

\section{To cite this version:}

Denis Efimov, Tarek Raissi, Stanislav Chebotarev, Ali Zolghadri. Interval State Observer for Nonlinear Time Varying Systems. Automatica, 2013, 49 (1), pp.200-205. 10.1016/j.automatica.2012.07.004 . hal-00719911

\section{HAL Id: hal-00719911 \\ https://inria.hal.science/hal-00719911}

Submitted on 22 Jul 2012

HAL is a multi-disciplinary open access archive for the deposit and dissemination of scientific research documents, whether they are published or not. The documents may come from teaching and research institutions in France or abroad, or from public or private research centers.
L'archive ouverte pluridisciplinaire HAL, est destinée au dépôt et à la diffusion de documents scientifiques de niveau recherche, publiés ou non, émanant des établissements d'enseignement et de recherche français ou étrangers, des laboratoires publics ou privés. 


\title{
Interval State Observer for Nonlinear Time Varying Systems
}

\author{
Denis Efimov, Tarek Raïssi, Stanislav Chebotarev, Ali Zolghadri
}

\begin{abstract}
This paper is devoted to design of interval observers for Linear Time Varying (LTV) systems and a class of nonlinear time-varying systems in the output canonical form. An interval observer design is feasible if it is possible to calculate the observer gains making the estimation error dynamics cooperative and stable. It is shown that under some mild conditions the cooperativity of an LTV system can be ensured by a static linear transformation of coordinates. The efficiency of the proposed approach is demonstrated through numerical simulations.
\end{abstract}

\section{INTRODUCTION}

The problem of unmeasurable state vector estimation is very challenging and its solution is demanded in many applications [4], [5], [15]. In some situations due to presence of uncertainty (parametric or/and signal) the design of a conventional pointwise estimator, converging in the noise-free case to the ideal value of the state, is not possible. However, an interval estimation remains feasible. By interval or set-membership estimation we understand an observer that, using input-output information, computes an outer-approximation of the set of admissible values (interval) for the state at each instant of time. Another group of applications deals directly with evaluation of the set of admissible values for the state of an uncertain system (it can be the estimation goal in some fault detection systems, in biology or chemistry), the interval observers were proposed as a solution of this problem [6].

There are several approaches to design interval observers [3], [7], [8], [12]. This paper continues the framework of interval observer design based on the monotone system theory [3], [12]. That approach has been recently extended in [17] to nonlinear systems using Linear-Parameter-Varying (LPV) representation with known minorant and majorant matrices, and in [16] for observable nonlinear systems. One of the most complex assumptions for the interval observer design, dealing with cooperativity of the interval estimation error dynamics, was relaxed in [10], [16]. It was shown that under some mild conditions applying similarity transformation, a Hurwitz matrix could be transformed to Hurwitz and Metzler (cooperative). The transformation matrix is a solution of the Sylvester equation, a constructive procedure for this solution calculation was also given in [16].

The objective of this work is to develop the approach of interval observer design to the systems with non-constant matrices dependent on measurable input-output signals and time. In order to solve this problem an extension of the result from [16] is presented, that allows us to calculate a constant similarity transformation matrix representing a given interval of matrices to an interval of Metzler matrices. This result can be used to design interval observers for LPV systems with measurable vector of scheduling parameters [11], [18], [20] or LTV systems, that is the

The first author is with the Non-A project at INRIA - LNE, Parc Scientifique de la Haute Borne, 40 avenue Halley, Bât.A Park Plaza, 59650 Villeneuve d'Ascq, France, denis.efimov@inria.fr. The second author is with Conservatoire National des Arts et Métiers (CNAM), Département EASY, Cedric - laetitia 292, Rue St-Martin, case 2D2P10, 75141 Paris Cedex 03, tarek.raissi@cnam.fr. The third author is with the Department of Control Systems and Informatics, Saint Petersburg State University of Information Technologies Mechanics and Optics, Kronverkskiy av. 49, Saint Petersburg, 197101, Russia. The last author is with University of Bordeaux, IMS-lab, Automatic control group, 351 cours de la libération, 33405 Talence, France, Ali.Zolghadri@ims-bordeaux.fr. 
main novelty of the work. Two examples of such systems are considered in this work: LTV system and the Lorenz chaotic model (as a nonlinear system in the output canonical form).

The paper is organized as follows. Some basic facts from the theory of interval estimation are given in Section 2. The main result is described in Section 3. The examples of computer simulation are presented in Section 4.

\section{PRELiminaries}

Euclidean norm for a vector $x \in \mathbb{R}^{n}$ will be denoted as $|x|$, and for a measurable and locally essentially bounded input $u: \mathbb{R}_{+} \rightarrow \mathbb{R}\left(\mathbb{R}_{+}=\{\tau \in \mathbb{R}: \tau \geq 0\}\right)$ the symbol $\|u\|_{\left[t_{0}, t_{1}\right]}$ denotes its $L_{\infty}$ norm:

$$
\|u\|_{\left[t_{0}, t_{1}\right]}=e s s \sup \left\{|u(t)|, t \in\left[t_{0}, t_{1}\right]\right\}
$$

if $t_{1}=+\infty$ then we will simply write $\|u\|$. We will denote as $\mathcal{L}_{\infty}$ the set of all inputs $u$ with the property $\|u\|<\infty$. Denote the sequence of integers $1, \ldots, k$ as $\overline{1, k}$. The symbols $I_{n}$ and $E_{n}$ denote the identity matrix and the matrix with all elements equal 1 respectively (with dimension $n \times n$ ). For a matrix $A \in \mathbb{R}^{n \times n}$ the vector of its eigenvalues is denoted as $\lambda(A),\|A\|_{\max }=\max _{i=\overline{1, n}, j=\overline{1, n}}\left|A_{i, j}\right|$ (the elementwise maximum norm, it is not sub-multiplicative) and $\|A\|_{2}=\sqrt{\max _{i=\overline{1, n}} \lambda_{i}\left(A^{\mathrm{T}} A\right)}$ (the induced $L_{2}$ matrix norm), the relation

$$
\|A\|_{\max } \leq\|A\|_{2} \leq n\|A\|_{\max }
$$

is satisfied between these norms.

For two vectors $x_{1}, x_{2} \in \mathbb{R}^{n}$ or matrices $A_{1}, A_{2} \in \mathbb{R}^{n \times n}$, the relations $x_{1} \leq x_{2}$ and $A_{1} \leq A_{2}$ are understood elementwise. The relation $P \succ 0$ means that the matrix $P \in \mathbb{R}^{n \times n}$ is positive definite. Given a matrix $A \in \mathbb{R}^{m \times n}$ define $A^{+}=\max \{0, A\}, A^{-}=A^{+}-A$.

Lemma 1. Let $x \in \mathbb{R}^{n}$ be a vector variable, $\underline{x} \leq x \leq \bar{x}$ for some $\underline{x}, \bar{x} \in \mathbb{R}^{n}$, and $A \in \mathbb{R}^{m \times n}$ be a constant matrix, then

$$
A^{+} \underline{x}-A^{-} \bar{x} \leq A x \leq A^{+} \bar{x}-A^{-} \underline{x} .
$$

Proof: Note that $A x=\left(A^{+}-A^{-}\right) x$, that for $\underline{x} \leq x \leq \bar{x}$ gives the required estimates.

A matrix $A \in \mathbb{R}^{n \times n}$ is called Hurwitz if all its eigenvalues have negative real parts, it is called Metzler if all its elements outside the main diagonal are nonnegative. Any solution of the linear system

$$
\dot{x}=A x+\omega(t), \omega: \mathbb{R}_{+} \rightarrow \mathbb{R}_{+}^{n},
$$

with $x \in \mathbb{R}^{n}$ and a Metzler matrix $A$, is elementwise nonnegative for all $t \geq 0$ provided that $x(0) \geq 0$ [19]. Such dynamical systems are called cooperative (monotone) [19].

Lemma 2. [16] Given the matrices $A \in \mathbb{R}^{n \times n}, R \in \mathbb{R}^{n \times n}$ and $C \in \mathbb{R}^{p \times n}$. If there is a matrix $L \in \mathbb{R}^{n \times p}$ such that the matrices $A-L C$ and $R$ have the same eigenvalues, then there is a $S \in \mathbb{R}^{n \times n}$ such that $R=S^{-1}(A-L C) S$ provided that the pairs $\left(A-L C, e_{1}\right)$ and $\left(R, e_{2}\right)$ are observable for some $e_{1} \in \mathbb{R}^{1 \times n}, e_{2} \in \mathbb{R}^{1 \times n}$.

This result was used in [16] to design interval observers for linear time invariant systems with a Metzler matrix $R$ (the main difficulty is to prove the existence of a real matrix $S$, and to provide a constructive approach of its calculation). 


\section{MAIN RESULT}

In this work we consider the following model of a nonlinear time-varying system:

$$
\begin{gathered}
\dot{x}=A(t, y, u) x+f(t, x, u, \varrho), \\
y=C(t, u) x,
\end{gathered}
$$

where $x \in \mathbb{R}^{n}, u \in \mathbb{R}^{m}, y \in \mathbb{R}^{p}$ are the state, the input and the output of the system (3), $\varrho \in \Theta \subset \mathbb{R}^{q}$ is the vector of unknown signals or parameters, the compact set $\Theta$ is given, the matrix functions $A: \mathbb{R}^{p+m+1} \rightarrow \mathbb{R}^{n \times n}$, $C: \mathbb{R}^{m+1} \rightarrow \mathbb{R}^{p \times n}$ and the function $f: \mathbb{R}^{n+m+q+1} \rightarrow \mathbb{R}^{n \times m}$ are given. The instant values of $u(t) \in \mathcal{L}_{\infty}$, $y(t) \in \mathcal{L}_{\infty}$ are known. In this work we consider the case without measurement noise, the proposed result can be extended to the case with a noise in the measurement channel, this extension is omitted for brevity of presentation. Denoting $\theta(t)=\left[\begin{array}{lll}t & y & u\end{array}\right]^{\mathrm{T}}$ we can rewrite the system (3) in the quasi-LPV form with a measurable scheduling parameter vector $\theta$.

Many works on the interval observer design [3], [12], [17], [16] deal with the case of a constant matrix $A$ (or under some transformations the estimation error can be represented in the form with a constant matrix $A$, next an observer gain $L$ can be found such that $A-L C$ is Hurwitz and Metzler). In the present work, we are going to avoid such a restriction. First, we need the following assumptions.

Assumption 1. $\|x\| \leq X,\|u\| \leq U$ and $\|y\| \leq Y$, the constants $X>0, U>0$ and $Y>0$ are given.

Boundedness of the state $x$ and the input $u$ is a standard assumption in the estimation theory.

Assumption 2. Let $\underline{x} \leq x \leq \bar{x}$ for some $\underline{x} \in \mathbb{R}^{n}$ and $\bar{x} \in \mathbb{R}^{n}$, then $\underline{f}(t, \underline{x}, \bar{x}, u) \leq f(t, x, u, \varrho) \leq \bar{f}(t, \underline{x}, \bar{x}, u)$ for some given $\underline{f}: \mathbb{R}^{2 n+m+1} \rightarrow \mathbb{R}^{n}, \bar{f}: \mathbb{R}^{2 n+m+1} \rightarrow \mathbb{R}^{n}$ and all $t \geq 0,\|u\| \leq U, \varrho \in \Theta$.

Assumption 3. There exist matrix functions $L: \mathbb{R}^{p+m+1} \rightarrow \mathbb{R}^{n \times p}, P: \mathbb{R}_{+} \rightarrow \mathbb{R}^{n \times n}, P(\cdot)=P(\cdot)^{T} \succ 0$ such that for all $t \geq 0$ and $\|u\| \leq U,\|y\| \leq Y$ :

$$
\begin{gathered}
p_{1} I_{n} \preceq P(t) \preceq p_{2} I_{n}, p_{1}, p_{2}>0 ; \\
\dot{P}(t)+D(t, y, u)^{T} P(t)+P(t) D(t, y, u)+P(t)^{2}+Q \preceq 0, \\
D(t, y, u)=A(t, y, u)-L(t, y, u) C(t, u), Q=Q^{T} \succ 0 .
\end{gathered}
$$

Assumption 2 states that if the bounds $\underline{x}, \bar{x}$ on the state value $x$ are given, then the values of the nonlinear function $f$ are enclosed in the interval $[\underline{f}, \bar{f}]$ for all $\varrho \in \Theta$ (for a continuous $f$, the computation of $\underline{f}, \bar{f}$ for given $\underline{x}$, $\bar{x}$ and a convex $\Theta$ is a routine operation in the interval arithmetic [13]). In assumption 3 the observer gain $L(t, y, u)$ is introduced, that ensures stability of the time-varying matrix $D(t, y, u)$ with the Lyapunov function matrix $P(t)$, this assumption determines the generic stability conditions of the estimation dynamics. Due to assumption 1 the matrix $A$ is varying in a compact domain, then Linear Parameter-Varying or polytopic system results [1], [2], [14], [9] can be used to compute a gain $L$ satisfying assumption 3. If $D(t, y, u)=D(t)$, then this assumption is a conventional requirement for linear time-varying systems, if in addition $D(t)$ is periodical, then this inequality can be solved as a differential equation [21].

Under these assumptions, if we additionally assume that the matrix $D$ is Metzler, then the following interval observer can be designed [3], [12], [17]:

$$
\begin{aligned}
& \underline{\dot{x}}=A(t, y, u) \underline{x}+\underline{f}(t, \underline{x}, \bar{x}, u)+L(t, y, u)[y-C(t, u) \underline{x}], \\
& \dot{\bar{x}}=A(t, y, u) \bar{x}+\bar{f}(t, \underline{x}, \bar{x}, u)+L(t, y, u)[y-C(t, u) \bar{x}] .
\end{aligned}
$$


Theorem 1. Let assumptions $1-3$ hold, and the matrix $D(t, y, u)$ be Metzler for all $t \geq 0$ and $\|u\| \leq U,\|y\| \leq Y$. Let one of the following conditions be satisfied:

1) $|\underline{f}(t, \underline{x}, \bar{x}, u)|<+\infty,|\bar{f}(t, \underline{x}, \bar{x}, u)|<+\infty$ for any $t \geq 0,\|u\| \leq U$ and all $\underline{x} \in \mathbb{R}^{n}, \bar{x} \in \mathbb{R}^{n}$;

2) for any $t \geq 0,\|x\| \leq X,\|u\| \leq U$, $\varrho \in \Theta$ and all $\underline{x} \in \mathbb{R}^{n}, \bar{x} \in \mathbb{R}^{n}$

$$
\begin{gathered}
|f(t, x, u, \varrho)-\underline{f}(t, \underline{x}, \bar{x}, u)|^{2}+|\bar{f}(t, \underline{x}, \bar{x}, u)-f(t, x, u, \varrho)|^{2} \leq \\
\beta|x-\underline{x}|^{2}+\beta|\bar{x}-x|^{2}+\alpha
\end{gathered}
$$

for some $\alpha \in \mathbb{R}_{+}, \beta \in \mathbb{R}_{+}$, and

$$
\beta I_{n}-Q+R \preceq 0, R=R^{\mathrm{T}} \succ 0 .
$$

Then in (3), (4) the variables $\underline{x}(t)$ and $\bar{x}(t)$ remain bounded for all $t>0$ and

$$
\underline{x}(t) \leq x(t) \leq \bar{x}(t)
$$

provided that $\underline{x}(0) \leq x(0) \leq \bar{x}(0)$.

Proof: Consider the interval estimation errors $\bar{e}=\bar{x}-x, \underline{e}=x-\underline{x}$ :

$$
\begin{aligned}
& \dot{\dot{e}}=D(t, y, u) \underline{e}+f(t, x, u, \varrho)-\underline{f}(t, \underline{x}, \bar{x}, u), \\
& \dot{\bar{e}}=D(t, y, u) \bar{e}+\bar{f}(t, \underline{x}, \bar{x}, u)-f(t, x, u, \varrho) .
\end{aligned}
$$

Due to assumption 2 for a Metzler matrix $D$, for all $t \geq 0$ the properties $f[t, x(t), u(t), \varrho] \geq \underline{f}[t, \underline{x}(t), \bar{x}(t), u(t)]$, $\bar{f}[t, \underline{x}(t), \bar{x}(t), u(t)] \geq f[t, x(t), u(t), \varrho]$ and

$$
\underline{x}(t) \leq x(t) \leq \bar{x}(t)
$$

are satisfied, provided that $\underline{x}(0) \leq x(0) \leq \bar{x}(0)$. To prove that the variables $\underline{x}(t), \bar{x}(t)$ are bounded, consider the Lyapunov function $V=\underline{e}^{\mathrm{T}} P(t) \underline{e}+\bar{e}^{\mathrm{T}} P(t) \bar{e}$ and evaluate its derivative:

$$
\begin{aligned}
\dot{V}= & \underline{e}^{\mathrm{T}}\left[\dot{P}(t)+D(t, y, u)^{\mathrm{T}} P(t)+P(t) D(t, y, u)\right] \underline{e}+ \\
& \bar{e}^{\mathrm{T}}\left[\dot{P}(t)+D(t, y, u)^{\mathrm{T}} P(t)+P(t) D(t, y, u)\right] \bar{e}+ \\
& 2 \underline{e}^{\mathrm{T}} P(t)[f(t, x, u, \varrho)-\underline{f}(t, \underline{x}, \bar{x}, u)]+ \\
& 2 \bar{e}^{\mathrm{T}} P(t)[\bar{f}(t, \underline{x}, \bar{x}, u)-f(t, x, u, \varrho)] .
\end{aligned}
$$

Due to assumption 3 this equality can be rewritten as follows:

$$
\begin{gathered}
\dot{V} \leq-\bar{e}^{\mathrm{T}} Q \bar{e}-\underline{e}^{\mathrm{T}} Q \underline{e}+ \\
|f(t, x, u, \varrho)-\underline{f}(t, \underline{x}, \bar{x}, u)|^{2}+|\bar{f}(t, \underline{x}, \bar{x}, u)-f(t, x, u, \varrho)|^{2} .
\end{gathered}
$$

If the first condition of the theorem is true, then the terms $|f(t, x, u, \varrho)-\underline{f}(t, \underline{x}, \bar{x}, u)|$ and $|\bar{f}(t, \underline{x}, \bar{x}, u)-f(t, x, u, \varrho)|$ are bounded for any $t \geq 0,\|x\| \leq X,\|u\| \leq U, \varrho \in \Theta$ and all $\underline{x} \in \mathbb{R}^{n}, \bar{x} \in \mathbb{R}^{n}$. Thus the errors $\bar{e}, \underline{e}$ are bounded by the standard Lyapunov arguments, and so are the variables $\underline{x}, \bar{x}$ (from assumption 1 the state $x$ is bounded). If the second condition of the theorem holds, then this inequality becomes:

$$
\dot{V} \leq-\bar{e}^{\mathrm{T}} R \bar{e}-\underline{e}^{\mathrm{T}} R \underline{e}+\alpha,
$$

that implies boundedness of $\underline{x}, \bar{x}$ by the same arguments.

The result of Theorem 1 is based on the rather restrictive assumption that the matrix $D$ is Metzler. All other 
assumptions are rather common in the estimation theory (boundedness of the state $x$ and the input $u$ in assumption 1, existence of majorant functions for $f$ from assumption 2, existence of the observer gain $L$ with the corresponding Lyapunov matrix $P$ in assumption 3, Lipschitz continuity or boundedness of $\underline{f}, \bar{f}$ stated in the theorem). For a constant matrix $D$ this assumption is relaxed in Lemma 2, where it is shown that under conditions of assumption 3 (the matrix $D$ is Hurwitz) there exists a static real similarity transformation matrix $S$ with $S^{-1} D S$ being Hurwitz and Metzler. In our case $D(t, y, u)$ is a matrix variable, an extension of Lemma 2 for this case is developed below.

Lemma 3. Let $Z \in \Xi \subset \mathbb{R}^{n \times n}$ be a matrix variable satisfying the interval constraints $\Xi=\left\{Z \in \mathbb{R}^{n \times n}: Z_{a}-\Delta \leq\right.$ $\left.Z \leq Z_{a}+\Delta\right\}$ for some $Z_{a}^{T}=Z_{a} \in \mathbb{R}^{n \times n}$ and $\Delta \in \mathbb{R}_{+}^{n \times n}$. If for some constant $\mu \in \mathbb{R}$ and a diagonal matrix $\Upsilon \in \mathbb{R}^{n \times n}$ the Metzler matrix $R=\mu E_{n}-\Upsilon$ has the same eigenvalues as the matrix $Z_{a}$, then there is an orthogonal matrix $S \in \mathbb{R}^{n \times n}$ such that the matrices $S^{T} Z S$ are Metzler for all $Z \in \Xi$ provided that $\mu>n\|\Delta\|$ max.

Proof: Note that the matrices $Z_{a}$ and $R$ are symmetric by their definition. Thus there exist two orthogonal matrices $O_{Z} \in \mathbb{R}^{n \times n}, O_{R} \in \mathbb{R}^{n \times n}$ such that $O_{Z}^{\mathrm{T}} Z_{a} O_{Z}=O_{R}^{\mathrm{T}} R O_{R}$ (these matrices may be composed by eigenvectors of $Z_{a}$ and $R$ respectively), the matrices $O_{Z}$ and $O_{R}$ can be chosen to satisfy $\left\|O_{Z}\right\|_{2}=\left\|O_{R}\right\|_{2}=1$. Let $S=O_{Z} O_{R}^{\mathrm{T}}$ be another orthogonal matrix with $\|S\|_{2}=1$, then $R=S^{\mathrm{T}} Z_{a} S$. For any $Z \in \Xi$ we have $S^{\mathrm{T}} Z S=S^{\mathrm{T}}\left(Z_{a}+\Pi\right) S$ for a matrix $\Pi \in \mathbb{R}^{n \times n},-\Delta \leq \Pi \leq \Delta$, then

$$
S^{\mathrm{T}} Z S=R+S^{\mathrm{T}} \Pi S
$$

and using (1) we get

$$
\left\|S^{\mathrm{T}} \Pi S\right\|_{\max } \leq\left\|S^{\mathrm{T}} \Pi S\right\|_{2}=\|\Pi\|_{2} \leq n\|\Pi\|_{\max } \leq n\|\Delta\| \|_{\max } .
$$

All elements outside of the main diagonal in the matrix $R$ equal $\mu$, thus all elements outside of the main diagonal of the matrix $R+S^{\mathrm{T}} \Pi S$ are not negative if $\mu>n\|\Delta\|_{\text {max }}$.

The matrix $\mu E_{n}$ has one eigenvalue $\mu n$ and the rest equal zero, the matrix $R$ for $\Upsilon=\rho I_{n}$ with $\rho>\mu n$ is Hurwitz and Metzler. To apply this lemma assume that all its conditions are satisfied.

Assumption 4. Let $D(t, y, u) \in \Xi$ for all $t \geq 0,\|u\| \leq U$ and $\|y\| \leq Y$, where $\Xi=\left\{D \in \mathbb{R}^{n \times n}: D_{a}-\Delta \leq\right.$ $\left.D \leq D_{a}+\Delta\right\}$ for some $D_{a}^{T}=D_{a} \in \mathbb{R}^{n \times n}$ and $\Delta \in \mathbb{R}_{+}^{n \times n}$. Let for some constant $\mu>n\|\Delta\|_{\text {max }}$ and a diagonal matrix $\Upsilon \in \mathbb{R}^{n \times n}$ the Metzler matrix $R=\mu E_{n}-\Upsilon$ has the same eigenvalues as the matrix $D_{a}$.

Under this assumption there is an orthogonal matrix $S \in \mathbb{R}^{n \times n}$ such that the matrices $S^{\mathrm{T}} D(t, y, u) S$ are Metzler for all $D(t, y, u) \in \Xi$. Introduce new state variable $z=S^{\mathrm{T}} x$, then the system (3) can be rewritten in the new coordinates:

$$
\dot{z}=S^{\mathrm{T}} A(t, y, u) S z+\phi(t, z, u, \varrho)
$$

where $\phi(t, z, u, \varrho)=S^{\mathrm{T}} f(t, S z, u, \varrho)$. Using (2) we have the following relations for $x=S z$ :

$$
\begin{gathered}
\underline{x} \leq x \leq \bar{x}, \\
\underline{x}=S^{+} \underline{z}-S^{-\bar{z}}, \bar{x}=S^{+} \bar{z}-S^{-} \underline{z},
\end{gathered}
$$

where $\underline{z} \leq z \leq \bar{z}$ are the interval estimates for the variable $z$. Under assumption 2 with the substitution of $\underline{x}, \bar{x}$ calculated in (5) we obtain:

$$
\begin{gathered}
\underline{\phi}(t, \underline{z}, \bar{z}, u)=S^{+\mathrm{T}} \underline{f}(t, \underline{x}, \bar{x}, u)-S^{-\mathrm{T}} \bar{f}(t, \underline{x}, \bar{x}, u) \leq \\
\phi(t, z, u, \varrho) \leq S^{+\mathrm{T}} \bar{f}(t, \underline{x}, \bar{x}, u)-S^{-\mathrm{T}} \underline{f}(t, \underline{x}, \bar{x}, u)=\bar{\phi}(t, \underline{z}, \bar{z}, u) .
\end{gathered}
$$


In the new coordinates the interval observer takes form similar to (4):

$$
\begin{aligned}
\dot{\dot{z}}= & S^{\mathrm{T}} A(t, y, u) S \underline{z}+\underline{\phi}(t, \underline{z}, \bar{z}, u)+ \\
& S^{\mathrm{T}} L(t, y, u)[y-C(t, u) S \underline{z}], \\
\dot{\bar{z}}= & S^{\mathrm{T}} A(t, y, u) S \bar{z}+\bar{\phi}(t, \underline{z}, \bar{z}, u)+ \\
& S^{\mathrm{T}} L(t, y, u)[y-C(t, u) S \bar{z}] .
\end{aligned}
$$

Now we are in position to prove the following relaxed variant of Theorem 1.

Theorem 2. Let assumptions 1-4 hold. Let one of the following conditions be satisfied:

1) $|\underline{f}(t, \underline{x}, \bar{x}, u)|<+\infty$ and $|\bar{f}(t, \underline{x}, \bar{x}, u)|<+\infty$ for any $t \geq 0,\|u\| \leq U$ and all $\underline{x} \in \mathbb{R}^{n}, \bar{x} \in \mathbb{R}^{n}$;

2) for any $t \geq 0,\|x\| \leq X,\|u\| \leq U, \varrho \in \Theta$ and all $\underline{z} \in \mathbb{R}^{n}, \bar{z} \in \mathbb{R}^{n}$

$$
\begin{gathered}
|\phi(t, z, u, \varrho)-\underline{\phi}(t, \underline{z}, \bar{z}, u)|^{2}+|\bar{\phi}(t, \underline{z}, \bar{z}, u)-\phi(t, z, u, \varrho)|^{2} \leq \\
\beta|z-\underline{z}|^{2}+\beta|\bar{z}-z|^{2}+\alpha
\end{gathered}
$$

for some $\alpha \in \mathbb{R}_{+}, \beta \in \mathbb{R}_{+}$, and

$$
\beta I_{n}-S^{\mathrm{T}} Q S+R \preceq 0, R=R^{\mathrm{T}} \succ 0 .
$$

Then in (3), (5), (6) the variables $\underline{x}(t)$ and $\bar{x}(t)$ are bounded for all $t>0$ and

$$
\underline{x}(t) \leq x(t) \leq \bar{x}(t)
$$

provided that $\underline{z}(0) \leq z(0) \leq \bar{z}(0)$.

Proof: Consider the dynamics of the interval estimation errors $\bar{e}=\bar{z}-z, \underline{e}=z-\underline{z}$ :

$$
\begin{aligned}
& \dot{\bar{e}}=S^{\mathrm{T}} D(t, y, u) S \bar{e}+\bar{\phi}(t, \underline{z}, \bar{z}, u)-\phi(t, z, u, \varrho), \\
& \underline{\dot{e}}=S^{\mathrm{T}} D(t, y, u) S \underline{e}+\phi(t, z, u, \varrho)-\underline{\phi}(t, \underline{z}, \bar{z}, u) .
\end{aligned}
$$

Due to assumption 2 for a Metzler matrix $D$, for all $t \geq 0$ the properties $\phi[t, z(t), u(t), \varrho] \geq \underline{\phi}[t, \underline{z}(t), \bar{z}(t), u(t)]$, $\bar{\phi}[t, \underline{z}(t), \bar{z}(t), u(t)] \geq \phi[t, z(t), u(t), \varrho]$ and

$$
\underline{z}(t) \leq z(t) \leq \bar{z}(t)
$$

are satisfied, provided that $\underline{z}(0) \leq z(0) \leq \bar{z}(0)$. To prove that the variables $\underline{x}(t), \bar{x}(t)$ are bounded, consider the Lyapunov function $V=\underline{e}^{\mathrm{T}} S^{\mathrm{T}} P(t) S \underline{e}+\bar{e}^{\mathrm{T}} S^{\mathrm{T}} P(t) S \bar{e}$ derivative:

$$
\begin{aligned}
\dot{V}= & \underline{e}^{\mathrm{T}} S^{\mathrm{T}}\left[\dot{P}(t)+D(t, y, u)^{\mathrm{T}} P(t)+P(t) D(t, y, u)\right] S \underline{e}+ \\
& \bar{e}^{\mathrm{T}} S^{\mathrm{T}}\left[\dot{P}(t)+D(t, y, u)^{\mathrm{T}} P(t)+P(t) D(t, y, u)\right] S \bar{e}+ \\
& 2 \underline{e}^{\mathrm{T}} S^{\mathrm{T}} P(t) S[\phi(t, z, u, \varrho)-\underline{\phi}(t, \underline{z}, \bar{z}, u)]+ \\
& 2 \bar{e}^{\mathrm{T}} S^{\mathrm{T}} P(t) S[\bar{\phi}(t, \underline{z}, \bar{z}, u)-\phi(t, z, u, \varrho)] .
\end{aligned}
$$

Due to assumption 3 this equality can be rewritten as follows:

$$
\begin{gathered}
\dot{V} \leq-\bar{e}^{\mathrm{T}} S^{\mathrm{T}} Q S \bar{e}-\underline{e}^{\mathrm{T}} S^{\mathrm{T}} Q S \underline{e}+ \\
|\phi(t, z, u, \varrho)-\underline{\phi}(t, \underline{z}, \bar{z}, u)|^{2}+|\bar{\phi}(t, \underline{z}, \bar{z}, u)-\phi(t, z, u, \varrho)|^{2} .
\end{gathered}
$$

If the first condition of the theorem is true, then the terms $|\phi(t, z, u, \varrho)-\underline{\phi}(t, \underline{z}, \bar{z}, u)|$ and $|\bar{\phi}(t, \underline{z}, \bar{z}, u)-\phi(t, z, u, \varrho)|$ 
are bounded for any $t \geq 0,\|x\| \leq X,\|u\| \leq U, \varrho \in \Theta$ and all $\underline{z} \in \mathbb{R}^{n}, \bar{z} \in \mathbb{R}^{n}$. Thus the errors $\bar{e}, \underline{e}$ are bounded by the standard Lyapunov arguments, and so are the variables $\underline{z}, \bar{z}$. Due to (5) the same is true for the variables $\underline{x}$, $\bar{x}$. If the second condition of the theorem holds, then this inequality takes form:

$$
\dot{V} \leq-\bar{e}^{\mathrm{T}} R \bar{e}-\underline{e}^{\mathrm{T}} R \underline{e}+\alpha,
$$

that implies boundedness of $\underline{x}, \bar{x}$ by the same arguments.

This theorem proposes an interval observer for an LTV (LPV) system explicitly skipping the requirement on cooperativity of the closed loop matrix $D$. Indeed according to assumption 3 it is only stable and there exists a transformation of coordinates that makes the closed loop dynamics cooperative. To apply this theorem, if we have a solution $L$ from Assumption 3 ensuring the estimation error stability, then we may try to calculate $D_{a}$ and $\Delta$ for the obtained $D$ in order to verify that the transformation matrix $S$ exists. Next, the interval observer equations are given in (6).

\section{EXAMPLES}

To illustrate the proposed results let us consider two examples.

\section{A. Lorenz chaotic system}

Consider a variant of the Lorenz model:

$$
\begin{aligned}
& \dot{x}_{1}=\sigma\left(x_{2}-x_{1}\right), y=x_{1}, \\
& \dot{x}_{2}=-\eta x_{2}-x_{1} x_{3}+\varrho x_{1}, \\
& \dot{x}_{3}=-\beta x_{3}+x_{1} x_{2},
\end{aligned}
$$

where $\eta, \beta, \sigma$ are constant parameters assumed to be known, parameter $\varrho$ is time-varying and unknown, $\varrho_{\min } \leq$ $\varrho \leq \varrho_{\max }$ where $\varrho_{\max }>0$ and $\varrho_{\min }>0$ are given. The system can be represented in the form (3) using the designations:

$$
\begin{gathered}
A(y)=\left[\begin{array}{ccc}
-\sigma & \sigma & 0 \\
0 & -\eta & -y \\
0 & y & -\beta
\end{array}\right], f(y, \varrho)=\left[\begin{array}{c}
0 \\
\varrho y \\
0
\end{array}\right], \\
C=\left[\begin{array}{lll}
1 & 0 & 0
\end{array}\right] .
\end{gathered}
$$

We will assume that assumption 1 holds for the chosen parameters and initial conditions. Obviously the assumption 2 is satisfied for

$$
\bar{f}(y)=\left\{\begin{array}{lll}
\varrho_{\max } y & \text { if } & y>0 \\
\varrho_{\min } y & \text { if } & y \leq 0
\end{array}, \underline{f}(y)=\left\{\begin{array}{lll}
\varrho_{\min } y & \text { if } & y>0 \\
\varrho_{\max } y & \text { if } & y \leq 0
\end{array},\right.\right.
$$

since $y$ is bounded, these limiting functions are also bounded and the first condition of theorems 1 or 2 is verified. Direct computation shows that assumption 3 is true for $L=[0 \sigma 0]^{\mathrm{T}}$ and $P=I_{3}$. In assumption 4 we can choose

$$
D_{a}=\left[\begin{array}{ccc}
-\sigma & 0 & 0 \\
0 & -\eta & 0 \\
0 & 0 & -\beta
\end{array}\right], \Delta=\left[\begin{array}{ccc}
0 & \sigma & 0 \\
\sigma & 0 & y_{\max } \\
0 & y_{\max } & 0
\end{array}\right],
$$

where $y_{\max }$ is the maximal admissible amplitude of the output. 

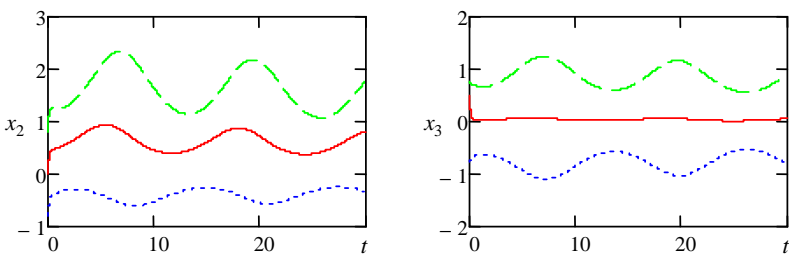

Figure 1. The results of simulation for Lorenz model for $\varrho_{\min }=8, \varrho_{\max }=12$
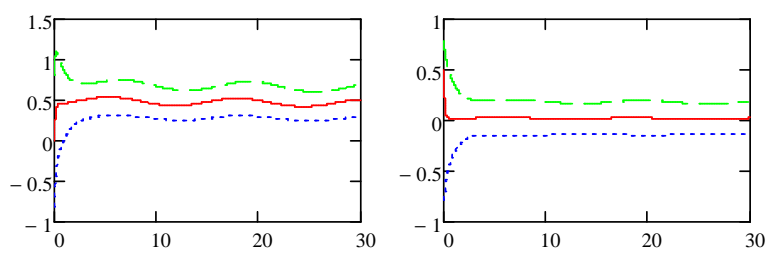

Figure 2. The results of simulation for Lorenz model for $\varrho_{\min }=9.5, \varrho_{\max }=10.5$

The following values of parameters are used for simulation:

$$
\sigma=1, \eta=10, \beta=10
$$

In addition two scenarios are considered:

$$
\begin{gathered}
\varrho_{\min }=8, \varrho_{\max }=12, \varrho(t)=10+2 \sin (0.5 t) ; \\
\varrho_{\min }=9.5, \varrho_{\max }=10.5, \varrho(t)=10+0.5 \sin (0.5 t) .
\end{gathered}
$$

For these values $y_{\max }=1$ and

$$
\begin{gathered}
R=\mu E_{n}-\Upsilon, \mu=3 y_{\max }, \Upsilon=10 I_{3}, \\
S=\left[\begin{array}{ccc}
0.577 & 0.577 & 0.577 \\
-0.816 & 0.735 & 0.082 \\
0 & -0.779 & 0.779
\end{array}\right] .
\end{gathered}
$$

The results of interval estimation for the unmeasured coordinates $x_{2}$ and $x_{3}$ are shown in figures 1 and 2. As we can conclude, the width of the estimated interval is proportional to the uncertainty size, i.e. $\varrho_{\max }-\varrho_{\min }$ in this example.

\section{B. LTV system}

Now consider the LTV system

$$
\dot{x}=A(t) x+b(t), y=x_{2},
$$

where $x \in \mathbb{R}^{2}$ is the state and

$$
A(t)=\left[\begin{array}{cc}
-0.632-0.8 \sin (t) & 0.5 \cos (3 t) \\
-0.7 \cos (2 t) & 0.3 \sin (t)
\end{array}\right],
$$



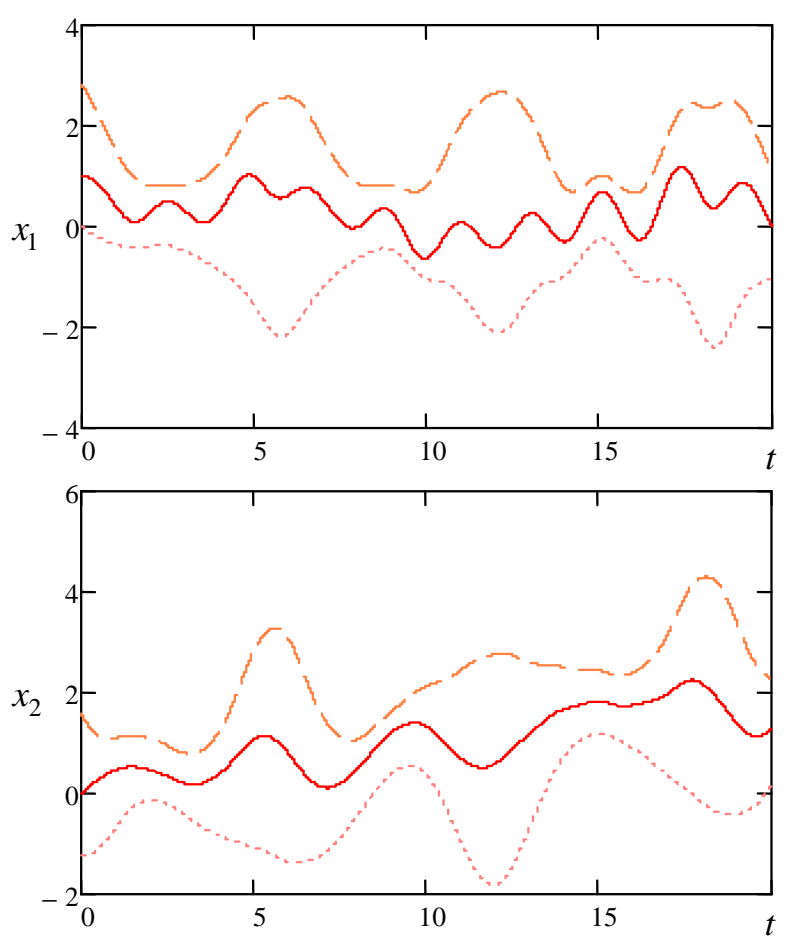

Figure 3. The results of simulation for LTV system

$b(t)$ is an uncertain input such that

$$
\left[\begin{array}{l}
-0.1 \\
-0.4
\end{array}\right]=\underline{b} \leq b(t) \leq \bar{b}=\left[\begin{array}{l}
0.3 \\
0.6
\end{array}\right],
$$

during simulation $b(t)=\left[\begin{array}{lll}0.1+0.2 \sin (0.5 t) & 0.1+0.5 \cos (1.5 t)\end{array}\right]^{\mathrm{T}}$ (assumption 2 holds). The matrix $A(t)$ is unstable and not Metzler, but on a finite time interval assumption 1 is satisfied. For $L=\left[\begin{array}{ll}0 & 4.368\end{array}\right]^{\mathrm{T}}$ the matrix $D(t)=A(t)-L C$ admits the requirements of assumption 3 with a periodical $0.01 I_{2} \leq P(t) \leq I_{2}$ and $Q=0.1 I_{2}$. Finally, the assumption 4 is satisfied for

$$
\begin{aligned}
D_{a} & =\left[\begin{array}{cc}
-0.632 & 0 \\
0 & -4.368
\end{array}\right], \Delta=\left[\begin{array}{ll}
0.8 & 0.8 \\
0.8 & 0.8
\end{array}\right], \\
R & =\left[\begin{array}{ll}
-2 & 1.8 \\
1.8 & -3
\end{array}\right], S=\left[\begin{array}{cc}
0.796 & -0.605 \\
0.605 & 0.796
\end{array}\right] .
\end{aligned}
$$

Therefore, all conditions of Theorem 2 are valid and the results of simulation of the obtained interval observer are presented in Fig. 3 for both coordinates. In this example the estimated interval width is also predefined by the model uncertainty $b(t)$.

\section{CONCLUSION}

The paper is devoted to an interval observer design for the LTV systems and the time-varying nonlinear systems in an output canonical form. It is the first time that the interval observer is designed for time-varying systems with a non-Metzler matrix $D$. A static transformation of coordinates is proposed mapping a stable LPV systems to another LPV system that is stable and cooperative. Thus the assumption that there exists an observer gain that 
makes the estimation error dynamics stable and cooperative is relaxed. The observer gain has to ensure stability of the estimation error as usual, next a static transformation of coordinates is proposed, that provides the required cooperativity. The efficiency is shown on examples of computer simulation.

The relaxation of symmetry of the matrix $D_{a}$ introduced in the conditions of the Lemma 3 and the stability conditions used in assumption 3 are the future directions of research.

\section{REFERENCES}

[1] Anstett F., Millerioux G., Bloch G. Polytopic observer design for LPV systems based on minimal convex polytope finding. J. Algorithms and Computational Technology, 3(1), 2009, pp. 23-43.

[2] Bara G.I., Daafouz J., Ragot J., Kratz F. State estimation for affine LPV systems. Proc. 39th IEEE Conf. Decision and Control, CDC, 2000, pp. 4565-4570.

[3] Bernard O., Gouzé J.L. Closed loop observers bundle for uncertain biotechnological models. J. Process Control, 14, 2004 , pp. 765-774.

[4] Control and Observer Design for Nonlinear Finite and Infinite Dimensional Systems. Lecture Notes in Control and Information Sciences, Vol. 322, Meurer T., Graichen K., Gilles E.-D. (Eds), Springer, 2005.

[5] Fossen T.I., Nijmeijer H. New Directions in Nonlinear Observer Design. Springer, 1999.

[6] Gouzé J.L., Rapaport A., Hadj-Sadok M.Z. Interval observers for uncertain biological systems. Ecological Modelling, 133, 2000, pp. $46-56$.

[7] Jaulin L. Nonlinear bounded-error state estimation of continuous time systems. Automatica, 38(2), 2002, pp. 1079-1082.

[8] Kieffer M., Walter E. Guaranteed nonlinear state estimator for cooperative systems. Numerical Algorithms, 37, 2004 , pp. 187-198.

[9] Köse I.E., Jabbari F. Control of LPV Systems with Partly Measured Parameters. IEEE Trans. Automatic Control, 44(3), 1999, pp. $658-663$.

[10] Mazenc F., Bernard O. Interval observers for linear time-invariant systems with disturbances. Automatica, 47(1), 2011 , pp. 140-147.

[11] Marcos A., Balas J. Development of linear-parameter-varying models for aircraft. J. Guidance, Control, Dynamics, $27(2), 2004$.

[12] Moisan M., Bernard O., Gouzé J.L. Near optimal interval observers bundle for uncertain bio-reactors. Automatica, 45(1), 2009, pp. 291-295.

[13] Moore R.E., Kearfott R.B., Cloud M.J. Introduction to Interval Analysis. Philadelphia, SIAM, 2009.

[14] Ichalal D., Marx B., Ragot J., Maquin D. An approach for the state estimation of Takagi-Sugeno models and application to sensor fault diagnosis. Proc. 48th IEEE Conf. Decision and Control, CDC/CCC, 2009, pp. 7789-7794.

[15] Nonlinear Observers and Applications. Lecture Notes in Control and Information Sciences, Vol. 363, Besançon G. (Ed.), Springer, 2007.

[16] Raïssi T., Efimov D., Zolghadri A. Interval state estimation for a class of nonlinear systems. IEEE Trans. Automatic Control, 57(1), 2012, pp. 260-265.

[17] Raïssi T., Videau G., Zolghadri A. Interval observers design for consistency checks of nonlinear continuous-time systems. Automatica, 46(3), 2010, pp. 518-527.

[18] Shamma J., Cloutier J. Gain-scheduled missile autopilot design using linear parameter-varying transformations. J. Guidance, Control, Dynamics, 16(2), 1993, pp. 256-261.

[19] Smith H.L. Monotone Dynamical Systems: An Introduction to the Theory of Competitive and Cooperative Systems, vol. 41 of Surveys and Monographs, AMS, Providence, 1995.

[20] Tan W. Applications of Linear Parameter-Varying Control Theory. PhD thesis, Dept. of Mechanical Engineering, University of California at Berkeley, 1997.

[21] Tornambe A., Valigi P. Asymptotic stabilization of a class of continuous-time linear periodic systems. Systems and Control Letters, 28(4), 1996, pp. 189-196. 